\title{
Extended Standard Map with Spatio-Temporal Asymmetry
}

\author{
Taksu Cheon ${ }^{1}$, Pavel Exner ${ }^{2,3}$, and Petr Šeba ${ }^{3,4}$ \\ 1 Laboratory of Physics, Kochi University of Technology, Tosa Yamada, Kochi 782-8502, Japan \\ 2 Nuclear Physics Institute, Czech Academy of Sciences, 25068 Řež u Prahy, Czech Republic \\ 3 Doppler Institute, Czech Technical University, Břehová 7, 11519 Praha, Czech Republic \\ 4 Department of Physics, University of Hradec Kralove, 50003 Hradec Kralove, Czech Republic
}

(Dated: March 3, 2002)

\begin{abstract}
We analyze the transport properties of a set of symmetry-breaking extensions of the ChirikovTaylor Map. The spatial and temporal asymmetries result in the loss of periodicity in momentum direction in the phase space dynamics, enabling the asymmetric diffusion which is the origin of the unidirectional motion. The simplicity of the model makes the calculation of the global dynamical properties of the system feasible both in phase space and in controlling-parameter space. We present the results of numerical experiments which show the intricate dependence of the asymmetric diffusion to the controlling parameters.

KEYWORDS: deterministic diffusion, chaotic transport, molecular motor
\end{abstract}

PACS numbers: $5.40 . \mathrm{Fb}, 5.45 .-\mathrm{Gg}, 5.60 . \mathrm{Cd}, 87.15 . \mathrm{Aa}$

\section{INTRODUCTION}

The motion of a particle in temporally and spatially periodic potential has attracted renewed attention as a model of molecular motors [1]. In order to make it a motor, that generates unidirectional motion out of periodic motion, the potential has to be a "ratchet", i.e. its reflection symmetry in both spatial and temporal direction has to be broken, a fact generally known as a Curie's theorem. Traditionally, the friction has supplied the source of temporal symmetry breaking [2, 3, 4, 5. There are several recent works [6, 7] in which the frictionless "inertial" regime is studied where the time-asymmetric perturbation is the driving force of the unidirectional motion. Through these studies, it has become evident that the characeristics of the phase space plays a dicisive role. The exploration of the full phase space of a dynamical system, however, requires considerable computational resources. This naturally leads to models of ratchet dynamics in which the differential equation governing the time evolution is reducible to the discrete difference equations, namely the map [7]. One would then like to have a model of a system with ratchet dynamics, which is free from ad hoc assumptions and parameters that tend to obscure the underlying simple physics.

In this article, we propose a simple model of a particle motion under temporally and spatially periodic potential whose key feature is in that the obtained descrete map is a natural extention of the Chirikov-Taylor map, also known as the standard map [8] whose dynamical properties have been thouroughly analyzed. The unidirectional motion of the system is understood in the context of the deterministic diffusion found in the standard map. Also, as a practical benefit of the discrete evolution of the map dynamics, the computational burden is substantially reduced, and it becomes possible to study, for the first time, the transportation properties of a system in a broad region in the controlling parameter space. A very intricate dependence of the unidirectional motion on the control- ling parameter is revealed though a numerical investigation.

\section{EXTENDED STANDARD MAP: FRICTIONLESS CASE}

We consider the motion of a classical particle described by a time-periodic Hamiltonian with instantaneous potential action, or $\delta$-function kick, which has space periodic part and alternating-current type driving motion;

$$
H=\frac{p^{2}}{2}+\sum_{n=-\infty}^{\infty}\left[u(x)+x A s_{n}\right] \delta\left(t-n T\left\{1+\varepsilon s_{n}\right\}\right),
$$

where, for now, the trapping potential $u(x)$ is set to be symmetric and given by

$$
u(x)=-K \cos x,
$$

and $s_{n} \equiv(-1)^{n}$ is the parity of the iteration number $n$. The evolution of the system is described by

$$
\begin{aligned}
& \dot{x}=p \\
& \dot{p}=-\sum_{n=-\infty}^{\infty}\left[u^{\prime}(x)+A s_{n}\right] \delta\left(t-n T\left\{1+\varepsilon s_{n}\right\}\right),
\end{aligned}
$$

One can integrate the motion in between the $\delta$-function kicks and obtain the map which describe the evolution of position $x_{n}$ and momentum $p_{n}$ at discrete time $t_{n}=$ $n T\left(1+\varepsilon s_{n}\right)-0$

$$
\begin{aligned}
& x_{n+1}=x_{n}+p_{n+1}\left(1+\varepsilon s_{n}\right) \\
& p_{n+1}=p_{n}-K \sin x_{n}-A s_{n} .
\end{aligned}
$$

This set of equations can be regarded as a threeparameter extension of the standard map [9]. The parameter $K$, which is present in original standard map is the strength of the periodic potential that traps the 
particles in each periodic location. The parameter $A$ is the amplitude of alternating swing motion, and $\varepsilon$ is the measure of its time asymmetry. As with the case of the standard map, the dynamical properties of the system is studied through the analysis of phase space [10]. The transport property of standard map, $A=\varepsilon=0$, is characterized in terms of phase space average of functions of position and momentum $x$ and $p$. It is well known that the direct averages $\langle x\rangle$ and $\langle p\rangle$ are zeroas a direct result of the fact that the standard map is both time-reversal and space-reflectional symmetric. However the mean square $\left\langle p^{2}\right\rangle$ can be non zero when one has the chaotic motion unbounded by KAM tori in $p$-direction. This happens with sufficiently large value of $K$. One then has deterministic diffusion through chaotic motion which is characterized by the random-walk behavior, $\left\langle x^{2}\right\rangle \propto t$. In the extended standard map (5)-(6), a unidirectional motion can be realized if one has by the non-zero value for the $\langle p\rangle$ itself in the diffusive motion.

The reflection symmetries play an essential role in the existence (or non-existence) of the unidirectional motion [6]. One can rewrite (5)-(6) as

$$
\begin{aligned}
& -x_{n+1}=-x_{n}-p_{n+1}\left(1+\varepsilon s_{n}\right) \\
& -p_{n+1}=-p_{n}-K \sin \left(-x_{n}\right)+A s_{n} .
\end{aligned}
$$

These become identical in form to (5)-(6) themselves in case of $A=0$. Therefore, in this case, one has

$$
\begin{aligned}
& \left\langle x_{n}\right\rangle=\left\langle-x_{n}\right\rangle=0, \\
& \left\langle p_{n}\right\rangle=\left\langle-p_{n}\right\rangle=0 \quad(\text { for } A=0),
\end{aligned}
$$

which means that there cannot be any directed transport.

We now define $\tilde{x}_{n} \equiv x_{n}$ and $\tilde{p}_{n} \equiv p_{n+1}$, obtain a reverse map

$$
\begin{aligned}
\tilde{x}_{n-1} & =\tilde{x}_{n}-\tilde{p}_{n-1}\left(1-\varepsilon s_{n}\right) \\
-\tilde{p}_{n-1} & =-\tilde{p}_{n}-u\left(\tilde{x}_{n}\right)-A s_{n} .
\end{aligned}
$$

Therefore, if one sets $\varepsilon=0$, the map for $\left\{x_{n}, p_{n}\right\}$ and $\left\{\tilde{x}_{-n},-\tilde{p}_{-n}\right\}$ are identical, and one has

$$
\langle-p\rangle=\langle-\tilde{p}\rangle=\langle p\rangle=0 \quad(\text { for } \varepsilon=0)
$$

Therefore, one needs both $A \neq 0$ and $\varepsilon \neq 0$ to have directed motion. It is important to note that the map (5)-(6) in fact atcs differently for odd and ven $n$ because of the existence of $s_{n}$ term. It is advantageous to consider the phase spaces for odd $n$ and even $n$ separately. The reflection symmetry between (5)-([6) and (7)-(8) should then be interpreted as one between two phase space profiles each representing odd $n$ and even $n$.

For the standard map, $A=\varepsilon=0$, as is well known, the map is $2 \pi$-periodic in both $x$ and $p$ directions. The periodicity for $x$ direction is kept intact for non-zero $A$ and $\varepsilon$ : when one has a map $\left(x_{n}, p_{n}\right) \rightarrow\left(x_{n+1}, p_{n+1}\right)$, one also has

$$
\left(x_{n}+2 \pi, p_{n}\right) \rightarrow\left(x_{n+1}+2 \pi, p_{n+1}\right) .
$$
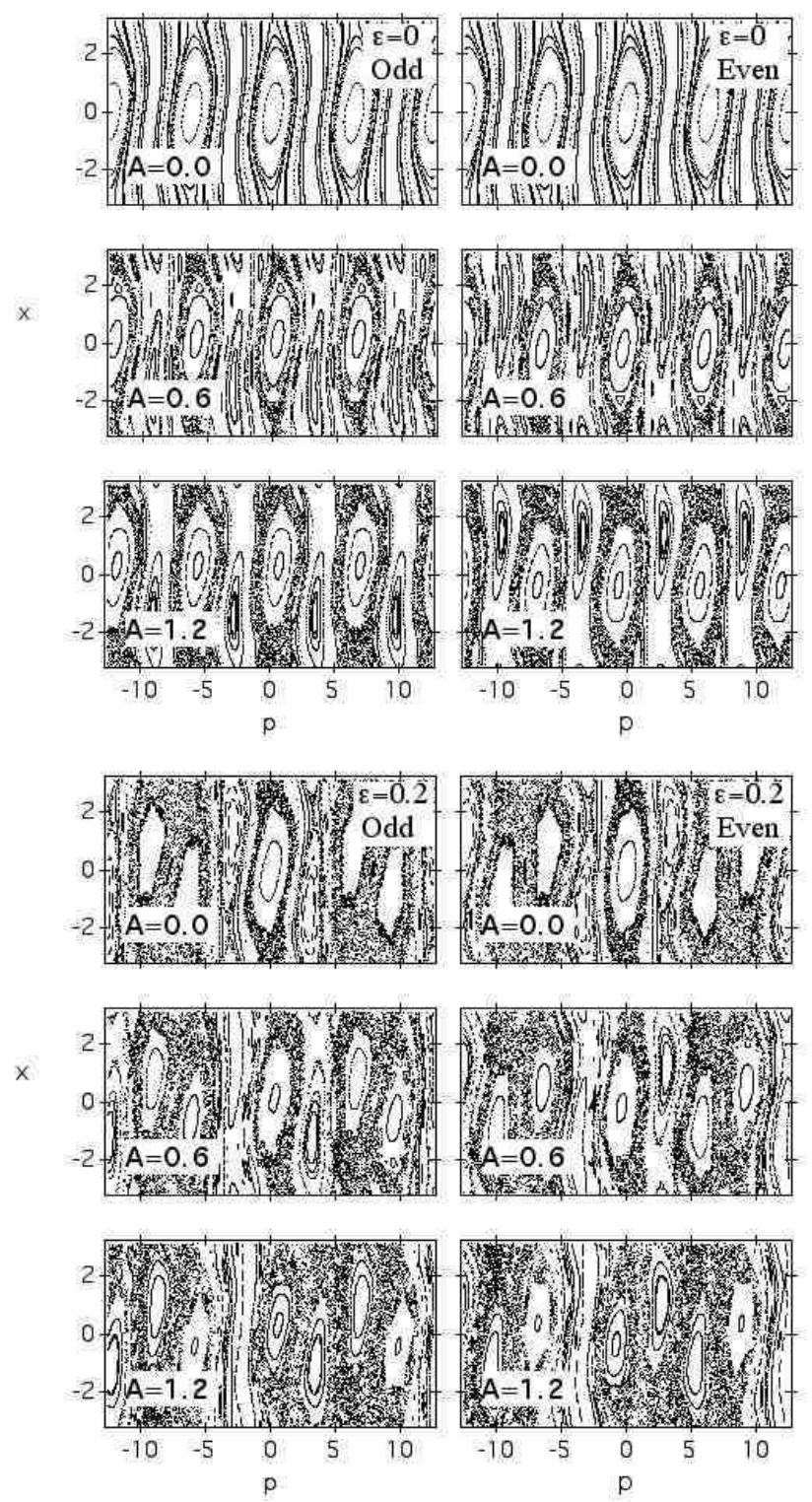

FIG. 1: Phase space profiles of the map Eqs. (5)-(6) for even numbers for $n$. The strength of periodic potential is set to be $K=0.6$. The temporal asymmetry parameter $\varepsilon$ and amplitude of alternating swing $A$ are varied. Left (right) column is for odd (even) $n$.

On the other hand, original $2 \pi$ periodicty for $p$ - direction is lost when non-zero $\varepsilon$ is present. If, however, $\varepsilon$ is a rational number, $M / N$ where $M$ and $N$ are mutually incomensurate positive integers, a new $2 N \pi$-periodicty for $p$-direction emerges, because in this case, one has

$$
\begin{aligned}
& \left(x_{n}, p_{n}+2 N \pi\right) \rightarrow \\
& \left(x_{n+1}+2(N \pm M) \pi, p_{n+1}+2 N \pi\right) \\
& \text { (for } \varepsilon=M / N ; N>M>0 \text { ). }
\end{aligned}
$$

The change of the $p$-periodicity has a direct impact on 

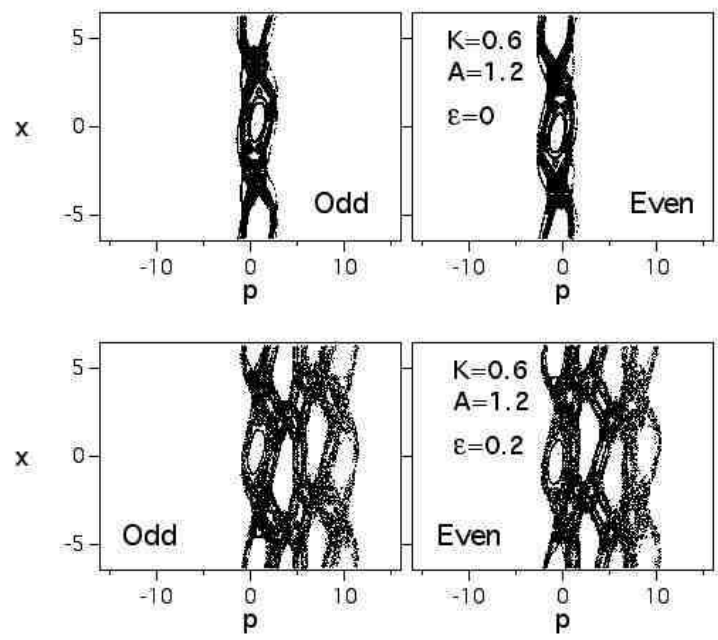

FIG. 2: Phase space orbits of the map (5)-(6) for $\varepsilon=0$ (top) and $\varepsilon=0.2$ (bottom) started from a set of random initial conditions confined in the region $|p|<\pi / 10$ and $|x|<\pi / 2$. The strength parameters are set to be $A=1.2$ and $K=0.6$. For the case of $\varepsilon=0$, one has $\langle p\rangle_{\text {odd }}+\langle p\rangle_{\text {even }}=0$.

the transportation properties of the extended standard map. The $2 N \pi$-periodicity in $p$-direction for a rational number $\varepsilon=M / N$ (where $M$ and $N$ are two mutually incommensurate natural numbers) means that the KAMtori (when they exit) appear at least once in $2 N \pi$ period. Thus the chaotic diffusion is bounded within the region in between two adjacent tori. This fact gives an estimate for the average momentum $\langle p\rangle$ within a region that include $p=0$ in the form

$$
\langle p\rangle \leq N \pi \quad(\text { for } \varepsilon=M / N ; N>M>0) .
$$

Note that only the upperbound is obtained since the exact location of the two adjacent KAM tori near $p=0$ cannot be determined a priori. This is evident when one considers $\varepsilon=0$ case, in which the map is $2 \pi$-periodic in $p$-direction, and a similar estimate to above gives $\langle p\rangle \leq$ $\pi$, while, as we have lernt in (12), it is zero because KAM tori is placed symmetrically around $p=0$.

The situation is illustrated in Fig.1 where we draw phase space profiles for the frictionless extende standard map with various $\varepsilon$ and $A$. The strength parameter is chosen to be $K=0.6$ in these examples. The effect of the breaking of phase space symmetries on the transportation properties of the system becomes evident by the inspection of Fig. 2, where we plot the portion of the phase space profile that is reached from a set of initial conditions clustered around in the vicinity of $p=0$. With the onset of non-zero $A$ and non-zero $\varepsilon$ terms, the average momenta in the chaotic region around $p=0$ is shifted from zero, and the directed tranport is realized. This is essentially in accordance with the conclusion of Flach et al. [6], in which they identify this phenomenon asymmetrical Levy flight. We are now ready to ready to look at the directed transport not just in one parameter
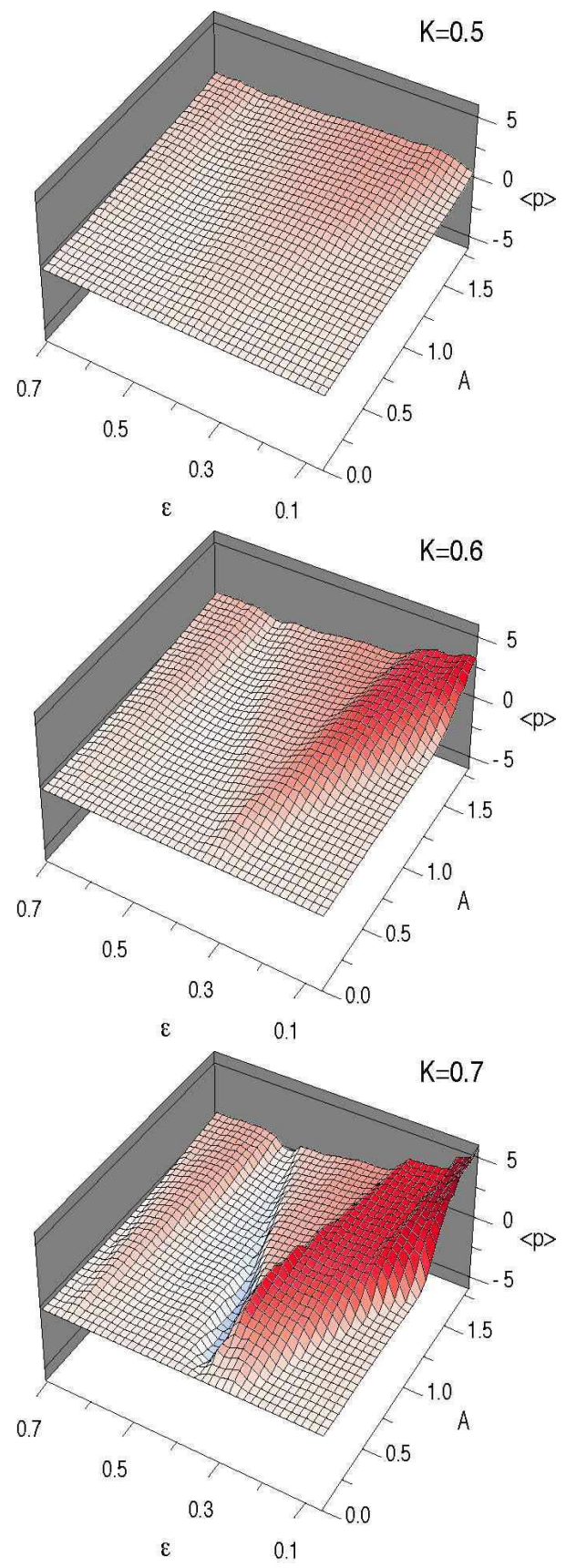

FIG. 3: A measure of directed motion $\langle p\rangle$ as the function of asymmetry parameter $\varepsilon$ and the strength of swing motion $A$ for the extended standard map with asymmetric kick (5)-(6)

set, but in a global region in the parameter space. In Fig. 3, we plot the average momentum $\langle p\rangle$ of the chaotic band that include $p=0$ as the function of swing parameter $A$ and time asymmetry $\varepsilon$. The coupling strength $K$ is taken to be $K=0.5$ (top), $K=0.6$ (middle) and $K=0.7$ (bottom). We start from a ensemble of 800 random initial configuration satisfying $|p|<\pi / 10$ and $|x|<$ $\pi / 2$. We symmetrize the ensemble in terms of $p-$ and $x-$ to guarantee $\langle p\rangle=0$ exactly at the start. Each config- 
uration is evolved with (5)-(5) for 12,000 times, and the momentum $p$ is averaged over that period and over the ensemble to obtain $\langle p\rangle$ for a given $A$ and $\varepsilon$. Calculation on the grid points of $48 \times 42$ in the space of $(A, \varepsilon)$ is used for each of the three figures.

From these figures, it appears that the direction of the asymmetric transport (positivity or negativity) is controllable, having step-like structure of increasing $\langle p\rangle$ with decreasing $\varepsilon$, which is in broad accordance to our arguments leading to (15). An intriguing feature is the direction-reversal of $\langle p\rangle$ with parameter variation, whose occurence is not at all obvious in the outset. Note also that the speed of unidirectional transport can be rather large: $\langle p\rangle$ is almost $2 \pi$ in the peak region, which means that the particle moves one spatial period in one temporal period.

\section{RATCHET MAP WITH FRICTION}

Our model can be extended to incorporate the ratchet dynamics with damping through the addition of friction. In place of eqs. (5)-(6), we set

$$
\begin{aligned}
\dot{x}= & p \\
\dot{p}= & -\sum_{n=-\infty}^{\infty}\left[u_{A S}^{\prime}(x)+A s_{n}\right] \\
& \times \delta\left(t-n T\left\{1+\varepsilon s_{n}\right\}\right)-\gamma p
\end{aligned}
$$

where we now add spatially asymmetric term to the trapping potential as

$$
u_{A S}^{\prime}(x)=K\left\{\sin x_{n}+2 \mu \sin \left(2 x_{n}+\delta\right)\right\} .
$$

The map obtained from these is written as

$$
\begin{aligned}
& x_{n+1}=x_{n}+p_{n+1}\left(e^{\gamma\left(1+\varepsilon s_{n}\right)}-1\right) / \gamma \\
& p_{n+1}=\left[p_{n}-u_{A S}^{\prime}\left(x_{n}\right)-A s_{n}\right] e^{-\gamma\left(1+\varepsilon s_{n}\right)}
\end{aligned}
$$

There are now six parameters in the model: $K, A, \mu, \delta$, $\gamma$, and $\varepsilon$. We reduce this number by setting $\varepsilon=0$. Since the temporal asymmetry is already broken by $\gamma$ alone, we do not expect to loose out much. We now obtain

$$
\begin{aligned}
& x_{n+1}=x_{n}+p_{n+1}\left(e^{\gamma}-1\right) / \gamma \\
& p_{n+1}=\left[p_{n}-u_{A S}^{\prime}\left(x_{n}\right)-A s_{n}\right] e^{-\gamma}
\end{aligned}
$$

As in the frictionless case, we consider the reflection symmetry of the map (21)-(22). With with the diffinition $\tilde{x}_{n} \equiv x_{n}$ and $\tilde{p}_{n} \equiv p_{n+1}$, one obtains a reverse map

$$
\begin{aligned}
\tilde{x}_{n-1} & =\tilde{x}_{n}-\tilde{p}_{n-1}(\gamma-1) / \gamma \\
-\tilde{p}_{n-1} & =-\tilde{p}_{n} e^{\gamma}+\left[-u_{A S}^{\prime}\left(\tilde{x}_{n}\right)-A s_{n}\right] e^{-\gamma}
\end{aligned}
$$

In the elastic limit $\gamma \rightarrow 0$, the map for $\left\{x_{n}, p_{n}\right\}$ and $\left\{\tilde{x}_{-n},-\tilde{p}_{-n}\right\}$ are identical, and one has

$$
\langle-p\rangle=\langle-\tilde{p}\rangle=\langle p\rangle=0 \quad(\text { for } \gamma \rightarrow 0)
$$
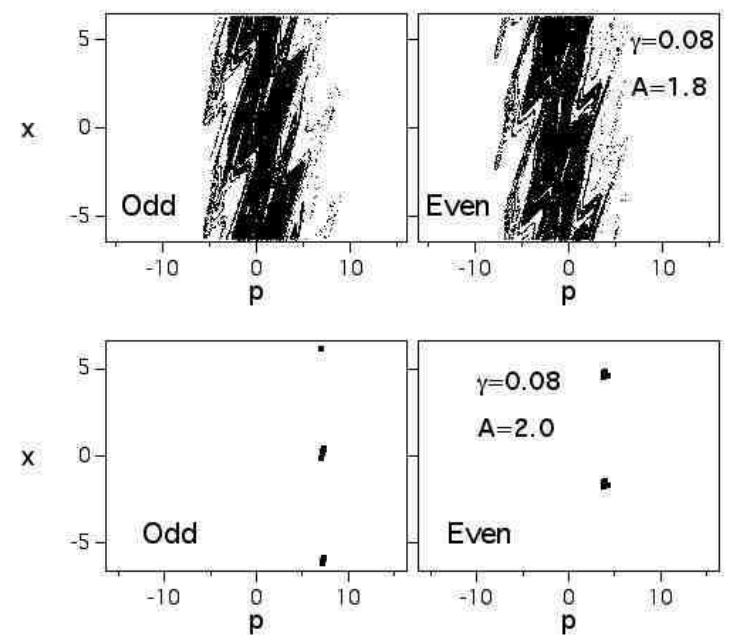

FIG. 4: Phase space orbit of map (21)-(22) after 10000 iteration showing the starnge attractor for $A=1.8$ (top figures), and the periodic attractor $A=2.0$ (bottom figures). Other parameters are set to be $\mu=0.2$ and $\delta=0.3$.

irrespective to the symmetry property of $u_{A S}$. This result, puzzling in first inspection, is of course in accordance with Curie's theorem. If, on the other hand, we have $A=0$, the motion is overdamped, and in sufficient steps, it will come to stand still, namely,

$$
x=x_{0} \quad\left(u_{A S}^{\prime}\left(x_{0}\right)=0\right), \quad p=0 \quad(\text { for } A=0)
$$

With friction, after sufficiently large steps $n$, all trajectories fall into the atractors. They can be either stable attractors with finite period, or the strange attractors with fractal dimension depending on the parameter value [11. Two examples from each case are shown as the top and bottom figures in Figs. 4. As is well known, these two cases alternate with minute variation of parameters. If one has $\mu=\delta=0$, the shape of attractor respects the spatial reflection symmetry, when both even and odd $n$ are considered. Therefore, one can only have $\langle p\rangle$ in this case. On the other hand, as long as $\mu \neq 0$ and $\delta \neq 0$, one has no spatial mirror symmetry in the phase space, and one should have $\langle p\rangle \neq 0$ in general. It is expected that the value of $\langle p\rangle$ can vary wildly as fuctions of parameter because of the afore-mntioned alternation of periodic and strange attractors with minute change of he parameter.

The transport property of the extended standard map with friction can be overviewd in Fig. 5, in which we show the three-dimensional plot of $\langle p\rangle$ as functions of two controlling parameters $A$ and $\gamma$. The spatial asymmetry parameters are fixed to be $\mu=0.2$ and $\delta=0.3$. The coupling strength $K$ is varied from $K=1.0$ to $K=1.2$ from the top to bottom. As before, we start from a $p-$ and $x$-symmetrized ensemble of 1600 initial configuration satisfying $|p|<\pi / 10$ and $|x|<\pi / 2$. Each configuration is evolved with (21)-(22) for 40,000 times, and the momentum $p$ is averaged over that period and over the ensemble to obtain $\langle p\rangle$ for a given $A$ and $\varepsilon$. The grid 

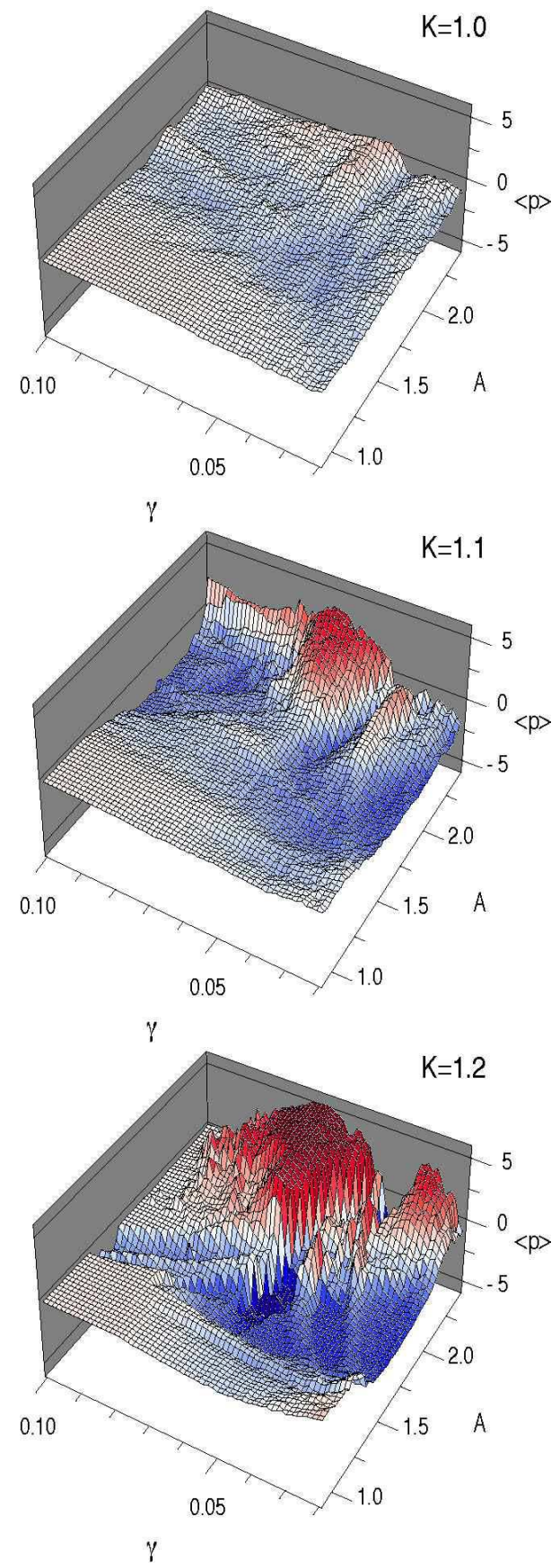

FIG. 5: The average $\langle p\rangle$ as the function of $\gamma$ and $A$ for the extended standard map with damping (21)-(22) The parameters controling the spatial asymmetry are set to be $\mu=0.2$ and $\delta=0.3$. points of $64 \times 64$ in the parameter space of $(A, \gamma)$ in the range $A \in[0.8,2.8]$ and $\gamma \in[0.02,0.10]$ are used for each of the three figures.

One can see from these figures, that the overdamped motion in ratchet potential shows directed transport, but is less controllable than the inertial counterpart, as expected from our preceeding arguments. At the moment, we do not have any clue as to the physical origin of the intriguing structure found in Fig. 5. However, we feel that the simplicity of our model (21)-(22) should yield to a mathematical analysis eventually to give us such clue.

\section{SUMMARY AND PROSPECTS}

In this work, an extended standard map which has spatial and temporal asymmetry is formulated. We have outlined its phase space properties, and have studied the unidirectional diffusion, which is thought to be relevant for molecular and biological motors. There exist several studies of great mathematical rigor on the standard map [12. 13. Our extended standard map may hopefully become a subject of similar research. One immediate extension of the current model is to introduce the randomness. Namely, one may consider $\varepsilon$ not as a fixed quantity, but a fluctuating number for each $n$. This is a simple way to include the thermal fluctuation into the model. Lastly, the study of quantum version of the current model may be of great interest. This is particularly so, because the quantum version of standard map [14, 15] is again a standard tool in the study of quantum chaotic diffusion.

\section{Acknowledments}

TC expresses his thanks to Prof. Izumi Tsutsui for helpful dicsussions. He also thanks members of the Theory Group of High Energy Accelerator Research Organization (KEK) at Tsukuba for the Hospitality extended to him during his stay. This work has been supported in part by the Grant-in-Aid (C) (No. 10640413) of the Japanese Ministry of Science and Education, and also by GAAS and the Czech Ministry of Education within the projects A1048101 and ME170.
[1] F. Julicher, A. Adjari and J. Prost, Rev. Mod. Phys. 69 (1997) 1269.

[2] R. Bartussek, P. Hänggi, J.G. Kissner, Euro. Phys. Lett 28 (1994) 459.

[3] P. Jung, J.G. Kissner, P. Hänggi, Phys. Rev. Lett 76 (1996) 3436.

[4] M. Porto, M. Urbakh, J. Klafter, Phys. Rev. Lett. 85
(2000) 491.

[5] M.N. Popescu, C.M. Arizmendi, A.L. Salas, F. Family, Phys. Rev. Lett. 85 (2000) 3321.

[6] S. Flach, O. Yevtushenko, Y. Zolotaryuk, Phys. Rev. Lett. 84 (2000) 2358.

[7] H. Schanz, M.-F. Otto, R. Ketzmerick and T. Dittrich, Phys. Rev. Lett. 87 (2001) 070601. 
[8] B.V. Chirikov, Phys. Rep. 52 (1979) 263

[9] T. Cheon, Bussei Kenkyu (Kyoto) 76 (2001) 248.

[10] See, for example, L.E. Reichl, The transition to chaos: in conservative classical systems: quantum manifestations (Springer, Heidelberg, 1991).

[11] E. Ott, Chaos in Dynamical systems (Cambridge Univ. Press, Cambridge, 1993).

[12] J.M. Greene J. Math. Phys. 20 (1979) 1183.
[13] R.S. MacKay, J.D. Meiss, Hamiltonian Dynamical Systems (Adam Hilger, Bristol, 1987).

[14] G. Casati, B. Chirikov, F. Izrailev, J. Ford, Leture Notes Phys. 93, ed. by G. casati and J. Ford (Springer, Berlin, 1979) p.334.

[15] S. Fishman, D.R. Grempel, R.E.Prange, Phys. Rev. Lett. 49 (1982) 509. 